\title{
El coAching COMO ESTRATEGIA PARA LA FORMACIÓN DE COMPETENCIAS PROFESIONALES
}

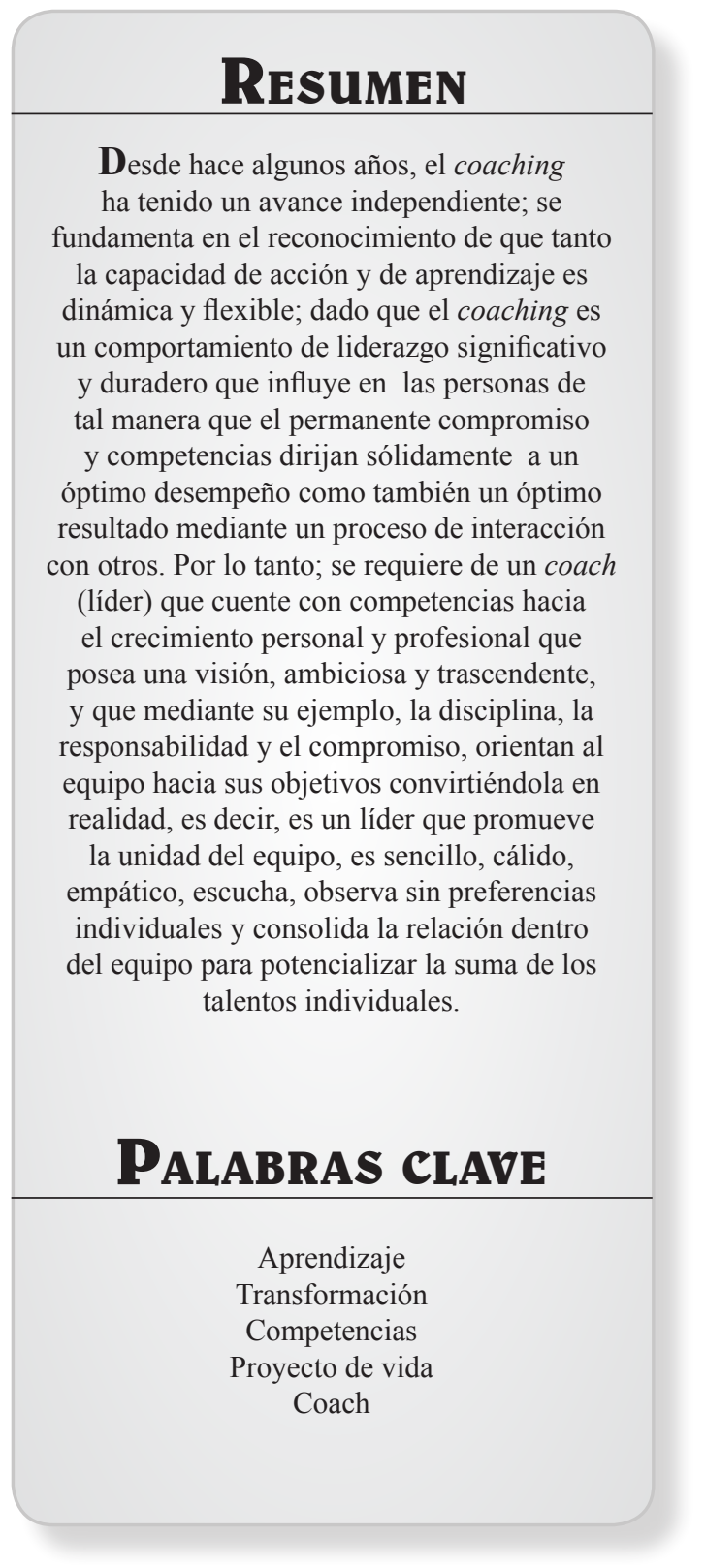

Luz Janeth Lozano Correa*

\section{Resumen}

Desde hace algunos años, el coaching ha tenido un avance independiente; se fundamenta en el reconocimiento de que tanto la capacidad de acción y de aprendizaje es dinámica y flexible; dado que el coaching es n comportamiento de liderazgo significativo y duradero que influye en las personas de tal manera que el permanente compromiso y competencias dirijan sólidamente a un optimo desempeño como también un óptim el crecimiento personaly profesional que posea una visión, ambiciosa y trascendente, y que mediante su ejemplo, la disciplina, la esponsabilidad y el compromiso, orientan a individuales y consolida la relación dentro talentos individuales.

Competencias

Proyecto de vida

Coach

\section{INTRODUCCIÓN}

$\mathbf{L}_{\text {o }}$ enfrentando por la globalización y la alta exigencia del entorno en los últimos años y la situación actual nos hacen sin duda ir en búsqueda de herramientas gerenciales que coadyuven a gestionar y liderar nuestros recursos físicos e intelectuales de manera eficiente procurando con esto encontrar la luz que nos direccione permitiendo menguar nuestras posibles carencias.

Podemos decir, que nos encontramos actualmente con una herramienta que seguramente nos puede contribuir mucho en nuestra gestión, potencializando nuestras competencias a través del ser; entendido lo anterior como el primer paso para hacer un estricto y ácido autoanálisis de fortalezas y oportunidades personales que se pongan en evidencia y pueda interiorizarlas tanto que logre resolverlas y simiente las bases para conseguir comprender e identificar entre los

\footnotetext{
* M.Sc. Gestión de Organizaciones. Especialista en Gerencia de Recursos Humanos, Psicóloga, docente investigadora, conferencista, consultora empresarial.
} 
talentos naturales propios y las cosas que puedo aprender; solo así cuando voy de adentro hacia afuera es que llego a encontrar mi verdadero yo profesional.

Es así como el coaching se ha convertido en una herramienta que aporta y contribuye al desarrollo de estrategias que favorecen el crecimiento personal y profesional de quienes se esfuerzan por conseguir proactivamente el éxito; por lo tanto, este puede entenderse como un proceso integral que busca ayudar a las personas a producir resultados extraordinarios en sus vidas, carreras, negocios y organizaciones; a través del cual se mejora el desempeño, se profundiza en el conocimiento de sí mismos y mejora la calidad de vida, proporcionando un aprendizaje que genera transformación de comportamientos sostenidos en el tiempo, con acciones y reflexiones continuas.

El coaching proviene de la palabra francesa coach que significa carruaje, es decir un vehículo para transportar personas de un lugar a otro. De hecho, la palabra sigue transmitiendo ese mismo significado en nuestros días. Un coach es literalmente un vehículo que lleva a una persona o a un grupo de personas de un origen a un destino deseado. El coaching es una herramienta, que ha sido usada desde hace varios años por países como Francia y Estados Unidos, y hoy en día ha tomado auge en España; y consiste en la creación de nuevos paradigmas con el fin de dar resultados innovadores a una necesidad dada, es decir que a través del uso del coaching existe la posibilidad de incrementar nuestro potencial al darnos cuenta de qué habilidades, herramientas y recursos disponemos con el fin de sobre pasar los obstáculos que nosotros mismo imponemos por el uso de paradigmas arcaicos que determinan nuestras actitudes y por consiguiente afecta nuestras aptitudes, en la medida que no somos

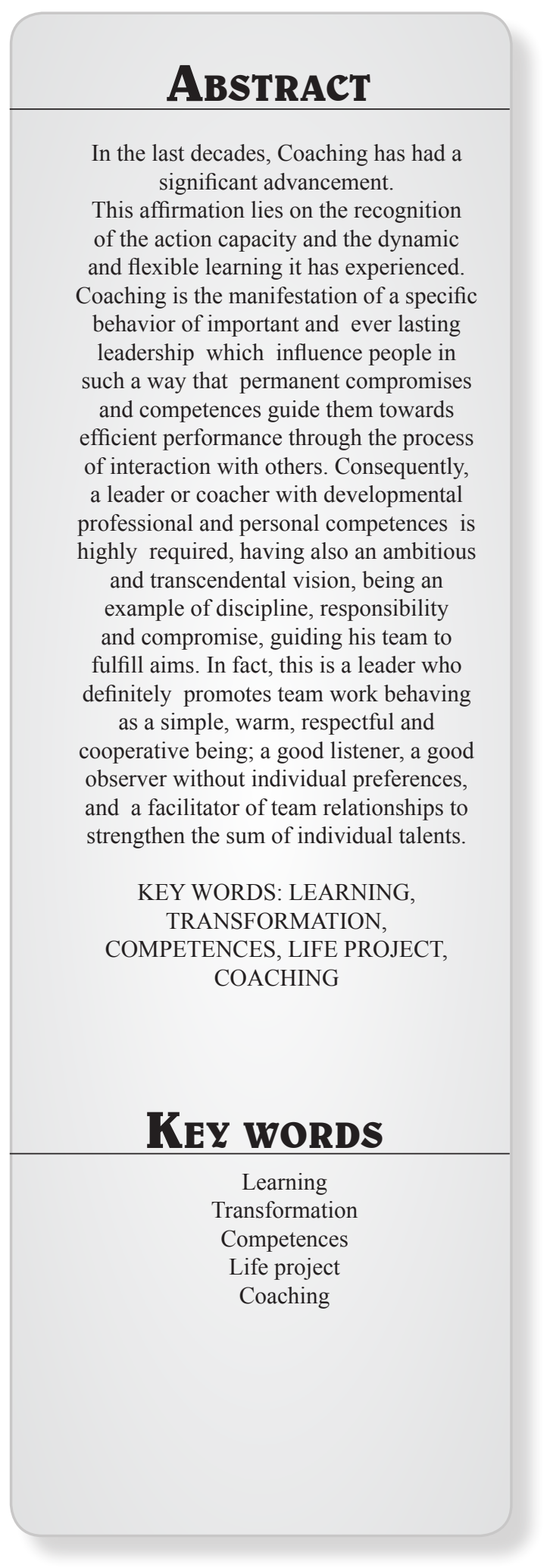


capaces de ponerlas en práctica posiblemente por miedo al cambio: además la carencia de una guía que nos muestre en qué estamos fallando y qué fortalezas tenemos puede facilitarnos el desarrollo de nuestras competencias.

Es así; como el coaching representa un medio estratégico para el siglo actual dado que el individuo busca hoy en día la superación personal con el propósito de ser más productivo y aprovechar la vida al máximo en sus diferentes contextos. Está basado en una relación donde el coach asiste en el aprendizaje de nuevas maneras de ser y de hacer, necesarias para generar un cambio paradigmático o cultural. El concepto de coach puede tomarse como un acompañamiento, o guía. En definitiva el coach no es más que un profesional "con formación especializada y conocimientos de técnicas de ayuda que permiten crear un espacio de diálogo entre él y el coachee (entrenado), un espacio de reflexión para mejorar". Por eso es tan fundamental que el profesional que quiera dedicarse al coach o acompañamiento profesional cuente por una parte con suficiente bagaje o experiencia y por otra con un considerable equilibrio personal y emocional. El coach se define como un profesional con amplia y reconocida experiencia que inspire confianza, que esté preparada a escuchar más que a hablar, y que pueda dar una retroalimentación constructiva, es comprensivo, integro, respetuosos y guarda la confidencialidad de todo, evidencia permanentemente su profesionalismo como también reconoce sus errores. Es un facilitador hacia nuevos aprendizajes y cambios estructurales. Los coach, NO están focalizados en el detectar errores, por el contrario, se basa en hacer que los individuos descubran por sí mismos sus fallas, con esta premisa cada uno aportará lo mejor de sí mismos para dar mejores rendimientos, un coach, lo que hace es indagar y trata de no hacer afirmaciones, y según su enfoque y su análisis podrá obtener resultados efectivos.

Entonces, sería importante preguntarnos como un coach puede contribuir al desarrollo de nuestras competencias personales y profesionales? Dado que es una persona que facilita cambios y es capacitada en relación que trabaja a los individuos a partir de sus competencias del ser, para fortalecer y hacer competitivo y productivo su desempeño a través del saber y hacer. Recordemos que el término de competencia se define como "el conjunto de conocimientos, actitudes y destrezas necesarias para desempeñar una ocupación dada", definición, heredera del concepto de McClelland (profesor de Psicología de Harvard) que es aparentemente sencilla pero que sintetiza algunas reflexiones y estudios complejos. En él observamos como el concepto de Competencia procede de la Psicología y se relaciona con el concepto de capacidad, ser capaz de realizar algo etc...Si consideramos esta definición enunciada, desde la perspectiva de la formación profesional, se destaca la idea de que para realizar con éxito una función determinada, se requieren conocimientos, actitudes y destrezas, lo cual requiere de coachs versados que fortalezcan con una dinámica flexible e innovadora coachees competentes y exitosos hacia nuevos retos.

Según Le Boterf (2001) experto en Ingeniería y Recursos Humanos, Competencia es la capacidad de movilizar y aplicar correctamente en un entorno laboral determinado recursos propios (habilidades, conocimientos y actitudes) y recursos del entorno para producir un resultado definido. Según esta definición; la competencia implica capacidad propia (habilidades) pero incluye la capacidad de movilizarla, además de movilizar los recursos del entorno, lo que supone una adaptación a 
cada situación, dotando al concepto de más complejidad para la inclusión efectiva al mercado laboral. Por lo tanto, se basa en la construcción de nuevos procesos fundamentados con un coach que se caracterice por: en comentarios asertivos, visión trascendente, que respeta profundamente a su grupo de trabajo, estimula el cambio y la transformación hacia nuevos aprendizajes.

Entonces, para desarrollar competencias no solo es necesario contar con un interés o motivación personal hacia el cambio, sino también con buenos orientadores 0 facilitadores que nos encausen hacia nuevos rumbos que provoquen una exigencia personal y técnica que me permita evidenciar $y / 0$ fortalecer mis talentos hacia el éxito profesional. Consecuentemente; el coaching es un proceso o medio que facilita este avance dado que para construir o mejorar competencias se debe considerar la conciencia de sí mismo; es decir, la comprensión de nosotros mismos y de cómo respondemos a nuestro entorno. Cuanta más conciencia tenga mas capacidad se tiene para entender a los demás y de interactuar mejor con ellos. Al conocernos mejor somos mas realistas de los cambios que requerimos y de poder equilibrar nuestros diferentes roles en diversos contextos, y así iniciamos el desarrollo de nuestras competencias del ser a partir de patrones de conducta como la introspección mediante una reflexión interna. Enseñar es el proceso consistente en ayudar a otros a desarrollar habilidades y capacidades. En el papel de maestro, el coach centra su atención sobre el juego interno de su cliente, apoyándole para que esté mentalmente preparado para dar lo mejor de sí. Así mismo, los buenos maestros ayudan a la persona a desarrollar nuevas estrategias para "aprender a aprender". La enseñanza se centra más en la adquisición de capacidades cognitivas generales que en formas concretas de actuar en situaciones específicas, los actuantes que han tenido éxito aprenden de sus errores, pero no se obsesionan con ellos. En relación con el coaching, los conceptos de enseñanza y desarrollo de capacidades se refieren a lo que podríamos denominar juego interno de la actuación. El concepto fue desarrollado por Timothy Gallwey $(1974,2000)$ como medio para ayudar a las personas a alcanzar la excelencia en diversos deportes (tenis, golf, esquí, etc.) en la música, en los negocios y en la formación empresarial. En cualquier área de actuación de que se trate, el éxito requiere la utilización tanto de la mente como del cuerpo. La esencia del juego interno consiste en prepararse mentalmente para actuar bien, es decir, está relacionado con nuestro enfoque mental a lo que estamos haciendo. Eso incluye la actitud, la seguridad en sí mismo y en el equipo, la capacidad para concentrarse eficazmente, tratar con las equivocaciones y con la presión para focalizar nuestro saber y hacer. El coaching se aplica en diversos campos: 


\section{Coaching personal ${ }^{1}$}

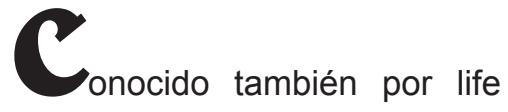
coaching es cuando el proceso de coaching se enfoca a aspectos personales del individuo. La finalidad del proceso es que el cliente adquiera el hábito de la pro actividad para que pueda alcanzar el nivel de resultados que desea en su encuentro consigo mismo.

El coaching es aplicable y viable en cada uno de los aspectos de nuestras vidas, a nivel empresarial es una herramienta altamente eficaz ya que desarrolla las capacidades de los empresarios a fin de crear en el gerente y equipo de trabajo una actitud de ganadores que contribuye al mejoramiento equilibrado de las actividades a desarrollar, produciendo así mayores ingresos a la compañía y una satisfacción personal al potencializar las habilidades de cada individuo integralmente.

\section{CoAching EMpresariaL}

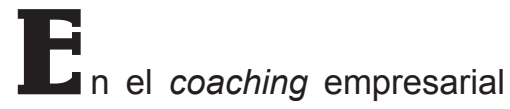

existen tres etapas:

Primera etapa: consiste en la ayuda de un coach a un coachee para plantear el problema (¿Qué?) Y analizar diferentes variables con el fin de hallar el punto de partida en busca de una solución a un problema.

Segunda etapa: es aquí donde se cuestionan los paradigmas que el coachee tiene con el propósito de crear una actitud más reflexiva para ayudarlo a encontrar nuevas formas de proceder. Es entonces en la segunda etapa donde se exige al coachee que derrumbe paradigmas que no permiten su desarrollo integral y siga modelos nuevos que faciliten su desempeño en la organización.
Tercera etapa: es el primer paso del coachee ya que es aquí cuando este puede dar una respuesta innovadora a la problemática que se presenta.

Hay que tener en cuenta que durante las tres etapas del coaching empresarial es esencial la ayuda mano a mano del coach, que proporcione una orientación efectiva. El Coaching Empresarial es una nueva disciplina inspirada en los grandes coach deportivos que es llevada al ámbito organizacional como una forma de desarrollar altas competencias y producir grandes saltos en el aprendizaje de gerentes y ejecutivos. Una de las fuentes del coaching empresarial es el llamado Coach Ontológico, que tiene su origen en las teorías filosóficas de Martín Heidegger y que

\footnotetext{
${ }^{1}$ Referencia Wikipedia-coaching personal.
} 
ha sido inicialmente desarrollada por Werner Erhard y seguida por Fernando Flores y Rafael Echeverría.

El ejecutivo o gerente -para convertirse en coach- requiere adquirir maestría en aquellos aspectos que más allá de sus competencias técnicas y gerenciales le habiliten como business coach en un contexto de liderazgo transformador de grupos humanos: esto es liderazgo de sistemas humanos configurados porseres humanos, cultural yemocionalmente interrelacionados, por múltiples y muy complejas redes conversacionales - verbales y no verbales- que intentan lograr objetivos individuales y colectivos. Un gerente, candidato a líder transportador, requiere tanto entender a fondo lo que esto significa como también aprender a ejercer influencia idealizada, generar motivación inspiradora, lograr estimulación intelectual y prestar consideración individualizada. De allí que al gerente para actuar como coach -en un contexto de liderazgo transformador- no le basta con incrementar sus competencias puramente lingüísticas o cognitivas- necesariamente perfectibles- sino que requiere además dominar competencias emocionales y corporales que le faciliten a él y a los grupos a cargo, alcanzar situaciones de óptimo desempeño individual, grupal y organizacional, conducentes al logro de elevados niveles de prosperidad y felicidad.

\subsection{Fundamentos del coaching empresarial}

Muchas compañías han comenzado a introducir los sistemas del coaching como estrategia para su competitividad global.
En el mundo de los negocios actual, solo aquellas empresas que estén comprometidas a innovar son las que podrán mantenerse competitivas en el mercado actual.

Se necesitan generar nuevos paradigmas que ayuden a crear una organización ganadora que incluya:

- Perfeccionamiento de una nueva filosofía de desarrollo humano.

- Creación de la transferencia de estrategias de aprendizaje.

- Utilización de los profesionales de desarrollo humano como consultores internos y responsables del desempeño de los sistemas gerenciales.

- Estímulo de las relaciones de los empleados creando una actitud de propietarios

- Utilización de los gerentes como coaches de desempeño.

- Creación de autoestima de los empleados y grupos.

- Identificación de estrategias de recompensa que motiven a los empleados a mejorar su compromiso y lograr resultados.

Para que pueda existir un coaching efectivo en las organizaciones, se debe tener en cuenta algunos aspectos intrínsecos al ser humano, ya que se debe entender que el coach es un ser humano con aciertos y debilidades.

Dentro de ellos se destaca:

\section{- Resultados}

El coaching es un proceso orientado a resultados y que tiene como consecuencia la mejora continua del desempeño, ya sea individual o grupal. 


\section{- Disciplina}

El coaching es una interacción disciplinaria. A fin de lograr la meta de la mejora continua, un coach debe ser lo suficientemente disciplinado como para crear las condiciones esenciales, aprender, desarrollar y utilizar las habilidades criticas y manejar adecuadamente una conversación de coaching.

\section{- Entrenamiento}

Para emprender conversaciones de coaching reales, se requiere de entrenamientos. No basta el conocimiento intuitivo o la simple memorización de ideas y conceptos, ya que esto no garantiza que se lleve a cabo conversaciones orientadas a mejorar el desempeño.

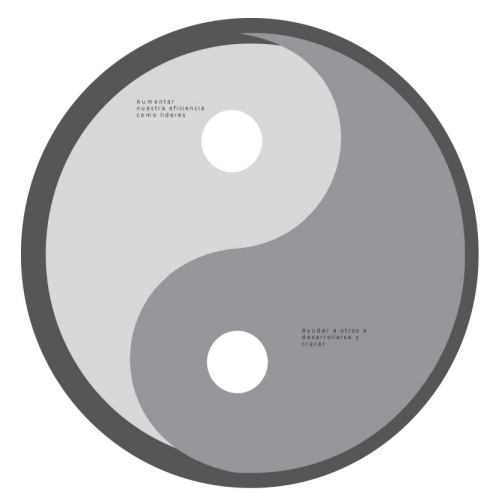

Fuente. www.laprimeraletra.com

\section{Coaching en la docencia}

$\mathbf{E}$ coaching para docentes es una estrategia que es usada hoy en día por lo general en España para contribuir al desempeño del docente en el aula de clase en diferentes aspectos que son importantes para poder guiar a un grupo de alumnos. Existe un libro dedicado a esta practica, coaching para docentes por Bou Pérez san Fernando, en el cual se plantea que el coaching es una buena herramienta con el fin de desarrollar habilidades en el docente aplicables a las aulas de clase tales como liderazgo, trabajo en equipo entre otras. La práctica del coaching en cualquier ámbito de nuestra vida es necesaria dado que nos guía aun cuando pensamos que estamos haciendo lo correcto, nos ubica en un plano más real en la vida y nos direcciona con el fin de despertar en nosotros la habilidad para ser mas creativos e innovadores con cada obstáculo que se nos presente.

En su articulo sobre Coaching Empresarial y en el desarrollo de la docencia, Ospina David, plantea que lo que se pretende de un coach profesional (en el caso que nos ocupa, alguien dedicado a la docencia), es que posea una serie de cualidades o habilidades que le permitan desarrollar su profesión con solvencia, entre la que destacamos: saber escuchar, ofrecer una disponibilidad, saber hacer su trabajo, ser competente, tener buen ánimo, una actitud mental positiva... y 
sobre todo una metodología precisa. El coachdocente, debido a la filosofía subyacente que envuelve a esta disciplina, no te dará consejos ni una solución ya hecha, simplemente ayudará al alumno a desarrollar sus propios recursos, a ser consciente de sus propias pistas, y a ser capaz de alcanzar los objetivos o las metas que se ha trazado. Además, un coach competente debe tener un conocimiento del entorno en el que tenga que intervenir sin tener la necesidad de ser un verdadero experto, a diferencia de lo que sería un consultor (especialista en el sector o ámbito donde actúa), una buena capacidad de relación, algunas nociones de psicología y/o desarrollo personal, y un perfecto conocimiento de si mismo, condición necesaria pero no suficiente para desarrollar una buena tarea.

A diferencia del terapeuta, que normalmente se adentra en el pasado para solucionar el presente, el coach trabaja entre el problema presente, bien delimitado, y el objetivo a la vista, suficientemente atractivo y realista para movilizar la motivación. Como dicen O'Connor y Larges, "si eres coach eres también líder, y el líder tiene tres atributos principales: habilidad, conocimiento y sirve de ejemplo. La habilidad como la dimensión que le permite "saber hacer", es capaz de actuar, de cambiar las cosas. El conocimiento como la dimensión que le permite "conocer", tiene capacidad de aprendizaje y una gran curiosidad por todo lo que le rodea. Y por último, es modelo para otras personas, desarrolla la dimensión del "ser". Se convierte cada vez más en la persona que quiere ser, se siente cómodo consigo mismo, tiene sus sueños, sus objetivos y sus valores y trabaja todos los días con ellos"?

\footnotetext{
${ }^{3}$ The International School of Coaching.
}

El buen docente, bajo los postulados del coaching se conoce a si mismo, tiene claros no sólo cuáles son sus objetivos sino también los valores y las creencias que los motivan, se ha trazado un plan de acción en el aula y siempre que puede es coherente: vive de acuerdo con sus valores y consistente: actúa y piensa de la misma manera. A partir de lo anterior; podemos considerar que el coaching independiente de su campo de acción requiere de coachs preparados y especializados en el desarrollo de sus competencias de saberhacer y ser, para generar cambios y nuevos aprendizajes que generen competitividad en nuestro día dio. Dentro del desarrollo de estas competencias se consideran ${ }^{3}$ :

- Generar conversaciones provocadoras:

- Escuchar lo que el cliente dice y no dice.

- Cuestionarse lo que se escucha.

- Hacer las preguntas adecuadas.

- Buscar claridad.

- Compartir con el cliente lo que sabes y como te sientes.

- Revelar el cliente a si mismo:

- Cuanto mejor se conoce uno a sí mismo, mejores decisiones puede tomar.

- Ayudar al cliente a descubrir sus dones, talentos, deseos, valores, necesidades y sueños, así como ayudarles a entender que es lo que les motiva e inspira.

- Resultado. Un cliente bien informado, que avanza rápidamente por el camino del auto-descubrimiento.

- Sacar grandeza

El Coach debe sacar a la superficie y desarrollar la grandeza del cliente, aumentando sus estándares. 
Disfrutar inmensamente del cliente

Disfrutar con el cliente = una aptitud

- Aptitud: cuando se disfruta con el cliente en su totalidad (en los momentos buenos y en los malos), se desarrolla un alto grado de confianza de forma natural.

- Beneficio: los clientes corren más riesgos y avanzan más rápidamente porque saben que el Coach esta completamente presente para ellos. Cuando la relación entre el Coach y el cliente llega a este punto, el Coaching es cooperativo y fácil, no pesado.

Ampliar los esfuerzos del cliente

- Ayudar a hacer más en menos tiempo, más de lo que harían los clientes solos.

- Actuar como catalizador y acelerador.

- Al ayudar al cliente a hacer más de lo que han hecho previamente o de lo que se creen capaces de hacer, aparece un importante valor añadido.

Navegar vía curiosidad

- El Coach es curioso por naturaleza, dejarse guiar por esa curiosidad.

- Beneficio: el verdadero beneficio de la curiosidad es que ambos, Coach y cliente, aprendan.

\section{- Reconocer la perfección en cada situación}

De lo que se trata aquí es de buscar y encontrar como un suceso, problema, situación - característica es perfecta, aún cuando claramente no lo es. Buscando comprender y reconocer la perfección primero, (en vez de ofrecer consejos, técnicas y soluciones de forma automática) es lo que el Coach hace de forma natural.

\section{- Poner rumbo a lo más importante}

Lo que es más importante para el cliente cambiará, es la naturaleza de los individuos que se encuentran en una fase de rápido crecimiento en sus vidas. El Coach es a la vez, rápido para identificar esa señal móvil y lo suficientemente flexible para ajustar el Coaching para ser efectivo en este nuevo camino.

- Comunicar claramente

Cuanto más clara sea la comunicación, menos se interpone en el camino de un gran Coaching, es por esto que debemos limpiar toda aquello que nos impida un buen estilo de comunicación, debemos empezar por eliminar ciertos prejuicios, juicios, necesidades insatisfechas, como "deberías", "podrías", discursitos, agendas, arrogancia y miedos.

\section{- Contar lo que percibes}

Los clientes confían en las observaciones e intuición del Coach para ayudarles a avanzar en la vida, así que cuanto más a menudo y más fácilmente un Coach pueda compartir lo que ve, siente y escucha, añade valor para ese cliente. A menudo, las indicaciones más sutiles y pequeñas son las que pueden actuar como poderosas guías y catalizadores en la vida o en el negocio del cliente. 
Ser el hincha del cliente

- Cuanto más a menudo, y más profundamente, el Coach se convierte en un hincha del cliente a todos los niveles (incluyendo sus acciones, progreso, sueños, características, compromisos, dones y cualidades), más apoyado se sentirá el cliente y mayores serán sus probabilidades de tener éxito.

- Para el Coach, simplemente respaldar no es suficiente, hay otro nivel mucho más elevado de apoyo que se genera cuando el Coach opera a nivel de hincha.

- Explorar nuevos territorios

- El Coach amplia la forma de pensar del cliente tejiendo nuevos conceptos, principios y distinciones durante la cesión de Coaching.

- Invita al cliente a experimentar con nuevos modelos, formas de hacer las cosas, e incluso identificar nuevas metas o resultados.

Saborear la verdad

- Siempre hay una verdad sobre una situación, una persona o un evento, que cuando se descubre y articula, puede transformar la vida o el negocio de una persona.

- Los Coaches han llegado al punto de disfrutar y orientarse entorno a la verdad como una fuente de alegría y de guía.
Diseñar un entorno favorable

- El éxito, por no mencionar la evolución personal, puede ser sostenible cuando existen entornos y estructuras de seguridad que lo apoyan.

- El Coach se entrena especialmente para ayudar al cliente a diseñar e instalar estos entornos.

- Respetar a la humanidad del cliente

- Respetar los límites tanto internos como externos.

- Reconociendo limites y respetando los distintos caminos al logro, el cliente es individual y universalmente respetado.

No podemos olvidar que debemos aprender a manejar nuestras influencias pasadas, presentes $y$ futuras; dadas que a partir de nuestra experiencia y proyecto de vida hacia lo que deseamos, debemos partir de nuestra honestidad, autenticidad, deseo y compromiso hacia el desarrollo personal y profesional con talento.

El coaching es un proceso que valora el bienestar y la autorrealización. Posee una doble via de ser y hacer. Por el lado del ser hace referencia en el cuidarse a uno mismo, la calidad de vida y el crecimiento como seres humanos, mientras que por el lado del hacer es un procedimiento de fomentar los rendimientos y un estilo de liderazgo que obtiene buenos resultados desde cualquier ámbito en que se aplique el coaching. Es decir; el bienestar del individuo es primordial y el desarrollo humano es el método esencial de obtener los mejores resultados. 


\section{Bibliografía}

Bradley J. (2007):Editorial Mac Graw Hill. Bogotá. El Coach de Negocios.

Ravier, L. (2005): Coaching como proceso.

Robert D. /2005): Coaching, Urano Ediciones.

Salazar Triviño G.(2005): Editorial Mc Graw Hill. Bogota. Coaching en acción.

Whitmore J. (2004):Editorial Paidos. Buenos Aires. COACHING EN ACCION.

Zeus P, Skiffington S. (2004): Coaching Práctico en el Trabajo. Ed. Mc Graw Hill.

The International School of Coaching, Barcelona España. www.internationalschoolofcoaching.

www.coach.com

www.eafit.edu.co

www.coachinghallinternational.com

www.aprendemas.com

instrumentos.Revista EAN, No. 47.

Conocimiento. Revista EAN. Na58 



\section{PAUTAS PARA LA PRESENTACIÓN DE ARTÍCULOS DESTINADOS A LA REVISTA INSTITUCIONAL DE LA UNIVERSIDAD EAN BOGOTÁ COLOMBIA}

\section{Elementos que debe incluir el artículo}

- Resumen en español y en inglés en el que se expliquen los objetivos específicos y las principales conclusiones del trabajo.

- Palabras claves en español y en inglés.

- Conclusiones o recomendaciones.

- Resumen de hoja de vida en máximo un párrafo, de cada uno de los autores.

- Datos de contacto de todos los autores. (email).

- Carta de autorización de la publicación del artículo, según el formato adjunto. En el caso de autores extranjeros o de otras ciudades, deben enviar el documento escaneado y donde se observen claramente las firmas de los mismos.

\section{Formato}

- Hojas tamaño carta $(21,5 \times 28 \mathrm{~cm}$.).

- Textos escritos en letra Arial 12, en procesador word.

- Espacio doble.

- Extensión mínima 15 cuartillas y máxima 25.

\section{Normas de presentación}

- Notas de pie de página breves con numeración correlativa al texto, pautas de citación correcta y completa bibliografía utilizada para la elaboración del escrito, ordenada alfabéticamente (Protocolo Harvard).

- Codificar figuras, tablas estadísticas, cuadros y gráficos de tal manera que al hacer mención de estos dentro del texto, la relación entre el escrito y el respectivo esquema sea coherente. Los dibujos, gráficos y curvas deben remitirse en tinta negra y en procesador de texto o en paquetes de diseño gráfico. Se deben enviar los archivos originales en los cuales fueron creadas las figuras y los gráficos.

- Concordancia entre los acápites, los textos y los cuadros, tablas, datos y figuras explicativas, adjuntando las series o variables, lo mismo que las notas de pie de página aclarativas, que deben observar la foliación. 
- Utilizar un sistema de nomenclatura que facilite la comprensión del texto.

Nota. Los trabajos presentados suponen que el material es original del autor y no ha sido publicado anteriormente o sometido simultáneamente a otra publicación.

\section{Naturaleza de los artículos}

Teniendo en cuenta que los artículos deben tener un carácter técnico, científico y cultural, el autor del texto puede considerar todos los temas; pero se dará prioridad a los artículos afines a las disciplinas del conocimiento a partir de las cuales la EAN genera sus programas de pregrado y postgrado y sus líneas de investigación.

Los artículos podrán ser resultados de investigaciones, estudios de casos, análisis teóricos donde se evidencien claramente los aportes analíticos del autos, etc.

\section{Sobre los derechos de autor}

- Los autores de artículos que se publiquen en la Revista, tendrán derecho a recibir 10 ejemplares del número de la edición en la que apareció la colaboración. El autor deberá autorizar a la EAN de manera escrita la publicación de su artículo.

- Los artículos publicados en la Revista de la EAN pueden ser reproducidos con fines académicos citando la fuente y el autor.

- Una vez aceptado un artículo para su publicación, el autor o autores transferirán los derechos a la Universidad EAN por medio de la firma de un Acta de sesión de derechos, de acuerdo con lo estipulado por la Dirección Nacional de Derechos de Autor de Colombia.

- En cualquier caso, el Comité Científico y Editorial de la Revista entiende que las opiniones vertidas por los autores son de su exclusiva responsabilidad.

\section{PASOS PARA LA EDICIÓN DE LA REVISTA}

- El editor recibe los artículos y los somete al Comité Editorial de la Revista.

- Los artículos seleccionados por el Comité Editorial serán evaluados por pares (evaluación doblemente ciega) externos quienes emitirán un concepto sobre la eventual publicación del artículo.

- La Universidad EAN se reserva el derecho de publicar o extractar los artículos y no se responsabiliza por el contenido de los mismos. 